Research Paper

\title{
De novo Assembly and Analysis of the Northern Leopard Frog Rana pipiens Transcriptome
}

\author{
Matthew K. Christenson ${ }^{1 \dagger}$, Andrew J. Trease ${ }^{1, \dagger}$, Lakshmi-Prasad Potluri ${ }^{1}$, Andrew J. Jezewski ${ }^{1, \dagger}$, Vincent M. \\ Davis $^{2}$, Lindsey A. Knight ${ }^{1}$, Alan S. Kolok ${ }^{1,3}$, Paul H. Davis ${ }^{1,4}{ }^{\bowtie}$ \\ 1. Department of Biology, University of Nebraska at Omaha, Omaha, Nebraska 68182, USA; \\ 2. Heteroskedastic, Inc., Arvada, Colorado 80403, USA; \\ 3. Center for Environmental Health and Toxicology, University of Nebraska Medical Center, Omaha, Nebraska 68198, USA; \\ 4. Department of Genetics, Cell Biology and Anatomy, University of Nebraska Medical Center, Omaha, Nebraska 68198, USA. \\ † Current addresses: MKC - Stowers Institute for Medical Research, Kansas City, Missouri 64110, USA. AJT - Department of Biochemistry \\ and Molecular Biology, University of Nebraska Medical Center, Omaha, Nebraska 68198, USA. AJJ -Department of Molecular Microbiology, \\ Washington University School of Medicine, St. Louis, Missouri 63110, USA
}

\section{$\triangle$ Corresponding author: pdavis@unomaha.edu.}

( ) Ivyspring International Publisher. This is an open-access article distributed under the terms of the Creative Commons License (http://creativecommons.org/ licenses/by-nc-nd/3.0/). Reproduction is permitted for personal, noncommercial use, provided that the article is in whole, unmodified, and properly cited.

Published: 2014.10.01

\begin{abstract}
The northern leopard frog Rana (Lithobates) pipiens is an important animal model, being used extensively in cancer, neurology, physiology, and biomechanical studies. R. pipiens is a native North American frog whose range extends from northern Canada to southwest United States, but over the past few decades its populations have declined significantly and is now considered uncommon in large portions of the United States and Canada. To aid in the study and conservation of $R$. pipiens, this paper describes the first $R$. pipiens transcriptome. The $R$. pipiens transcriptome was annotated using Gene Ontology (GO), Kyoto Encyclopedia of Genes and Genomes (KEGG), and Eukaryotic Orthologous Groups (KOG). Differential expression analysis revealed universal and tissue specific genes, and endocrine-related genes were identified. Transcriptome assemblies and other sequence data are available for download.
\end{abstract}

Key words: Northern Leopard Frog; Rana pipiens; Transcriptome.

\section{Results and Discussion}

\section{General Characteristics of the Rana pipiens Transcriptome}

To characterize the transcriptome of the northern leopard frog Rana (Lithobates) pipiens (Figure 1), a widely distributed North American species (Figure 2), cDNA samples were separately prepared from gonad, liver, kidney, brain, and tadpole homogenates and sequenced using paired-end $100 \mathrm{bp}$ reads [1]. The resulting 1.166 billion reads, comprising $116.6 \mathrm{~Gb}$ of sequence, were used to construct a transcriptome with Velvet and Oases (Table 1 and Table 2) [2,3]. Assembled transcriptomes and annotated characteristics are available at http://www.davislab.net/rana/, and raw reads have been deposited at NCBI under BioProject accession PRJNA240240.

\section{Organism Homology and Species Confirma- tion of Rana pipiens}

To identify transcript homologs, blastx was used to compare the $R$. pipiens transcriptome against the NCBI non-redundant (nr) protein database. Of the assembled transcripts, $91.7 \%(30,364)$ yielded significant BLAST hits. Among these, the western clawed frog Xenopus tropicalis and African clawed frog Xenopus laevis have the greatest number of top BLAST hits_(71.6\%). These result are consistent with accepted 
taxonomy but are strongly influenced by the completeness of protein annotation in the nr database; e.g. $X$. tropicalis is the closest, fully sequenced relative of $R$. pipiens. To further identify the completeness of the representative transcriptome, this set was compared via BLASTX against all Anura (frog) sequences in the nr database, resulting in more than $73 \%$ of the transcripts exhibiting a length coverage greater than $80 \%$ (Figure 3). To validate our transcriptome assembly as being from $R$. pipiens, we compared several genes (18S and $28 \mathrm{~S}$ rRNA, rhodopsin, and histone $\mathrm{H} 3$ ) to published sequences. The concatenated results indicate a significant identity $(99.42 \%)$ to previously published $R$. pipiens gene sequences, suggesting a preliminary level of genetic variation within the species (Table 3). Assembled sequence data also supported the previously published phylogenic differences between the Rana species established using these genes[4].

Table I. Information on sequencing reads for Rana pipiens.

\begin{tabular}{lll}
\hline Tissue & Number of Reads & Total Length \\
\hline Male Gonad & $32,771,881$ & $65,543,762 \mathrm{bp}$ \\
Male Liver & $35,791,829$ & $71,583,658 \mathrm{bp}$ \\
Female Gonad & $77,777,594$ & $155,555,188 \mathrm{bp}$ \\
Female Liver & $35,527,804$ & $71,055,608 \mathrm{bp}$ \\
Female Brain & $111,972,214$ & $223,944,428 \mathrm{bp}$ \\
Female Kidney & $108,243,825$ & $216,487,650 \mathrm{bp}$ \\
Tadpole & $181,032,472$ & $362,064,944 \mathrm{bp}$ \\
Total & $1,166,235,238$ & $116,623,523,800 \mathrm{bp}$ \\
\hline
\end{tabular}

The seven RNA samples, from both adult and juvenile R. pipiens tissues, were converted into individual libraries and were subsequently run on three sequencing lanes (Lane 1: Male Gonad, Male Liver, Female Gonad, Female Liver; Lane 2: Female Brain and Female Kidney; and Lane 3: Two Tadpole Stages) capturing 100 bp paired end reads.

Table 2. Statistics for Rana pipiens transcriptome assembly.

\begin{tabular}{lllll}
\hline $\begin{array}{l}\text { Number of Tran- } \\
\text { scripts }\end{array}$ & $\begin{array}{l}\text { Mean } \\
\text { Length }\end{array}$ & $\begin{array}{l}\text { Median } \\
\text { Length }\end{array}$ & N50 & $\begin{array}{l}\text { GC Con- } \\
\text { tent }\end{array}$ \\
\hline 33,086 & $2,639 \mathrm{bp}$ & $2,004 \mathrm{bp}$ & $3,783 \mathrm{bp}$ & 44.02 \\
\hline
\end{tabular}

The $R$. pipiens transcriptome was analyzed and the total number of transcripts, mean and median transcript length, N50, and GC content was determined.

Table 3. Percent identity to published Rana species sequences.

\begin{tabular}{ll}
\hline Species & Percent identity \\
\hline Rana pipiens & 99.42 \\
Rana chiricahuensis & 95.69 \\
Rana capito & 94.17 \\
Rana yavapaeniensis & 93.57 \\
Rana sylvatica & 90.57 \\
Rana temoparia & 89.5
\end{tabular}

Transcripts generated in this study were aligned to previously published Rana species sequences and a percent identity matrix was computed (4). This study utilized Rana_pipiens_Transcript_030065, 050213, 496664, and 023205 for 18s rRNA, histone $\mathrm{H} 3$, rhodopsin, and 28s rRNA sequences, respectively.

\section{Functional Annotation and Characterization of Rana pipiens Transcripts}

In an effort to both review the putative functions of the R. pipiens transcripts, and to validate the completeness of its transcriptome, multiple functional analyses were performed against $R$. pipiens and the $X$. tropicalis and X. laevis transcriptomes. Gene Ontology (GO) analysis was performed with $63.6 \%(21,056)$ of the transcripts being assigned GO terms (Figure 4). Importantly, $X$. tropicalis and $X$. laevis displayed a similar ontology pattern with the average percent difference in GO categories between R. pipiens and X. tropicalis of $25.03 \%$, as compared to X. tropicalis and X. laevis at $24.42 \%$ (calculated by summing the organism differences between each GO category, and dividing by the number of GO categories). Further examination using Eukaryotic Orthologous Groups (KOG) and Kyoto Encyclopedia of Genes and Genomes (KEGG) reveled that $59.8 \%(19,785)$ and $33.4 \%(11,064)$ of the transcripts were assigned KOG terms and K-numbers, respectively (Figure 5 and Figure 6). Overall, the nearly identical patterns observed in the GO, KOG, and KEGG analyses by R. pipiens, X. tropicalis, and X. laevis suggests the completeness of the $R$. pipiens transcriptome.

\section{Differential Expression of Transcripts in the Rana pipiens Tissues}

Differential expression analysis was carried out to determine the relative abundance of the $R$. pipiens transcripts within each tissue. In total, 23,058, 18,711, $16,359,18,325,24,960,27,247$, and 28,603 transcripts were detected and $72,12,76,33,182,612$, and 1,272 transcripts were unique to only that tissue in the male gonad, male liver, female gonad, female liver, female kidney, female brain, and tadpole, respectively (Figure 7). A file containing the relative expression of each transcript in individual tissues is available at http://www.davislab.net/rana/.

\section{Identification of Rana pipiens Endo- crine-Related Genes}

Increasing evidence demonstrates that agricultural contaminants, such as veterinary pharmaceuticals, fertilizers, and pesticides, can alter endocrine activities in wildlife and other vertebrates, including humans $[5,6]$. Therefore, because of its range and habitat, $R$. pipiens can serve as an ideal sentinel organism for monitoring the potential effects of these chemicals on other affected organisms. To this end, putative $R$. pipiens homologs of major endocrine-related genes were identified and exhibited a high degree of similarity to those genes of $X$. laevis (Table 4). 
Table 4. Rana pipiens endocrine-related genes.

\begin{tabular}{|c|c|c|c|c|c|c|c|}
\hline Gene Name & Gene Symbol & Gene ID & Query ID & $\begin{array}{l}\text { Length } \\
\text { (bp) }\end{array}$ & Subject ID & E-Value & $\begin{array}{l}\text { Bit } \\
\text { Score }\end{array}$ \\
\hline Androgen Receptor & ar & 399456 & Rana_pipiens_Transcript_250514 & 7358 & NP_001084353.1 & 0 & 1209 \\
\hline $\begin{array}{l}\text { Cytochrome P450, Family 17, Subfamily A, } \\
\text { Polypeptide } 1\end{array}$ & cyp17a1 & 100036774 & Rana_pipiens_Transcript_280688 & 2963 & AAG42003.1 & 0 & 777 \\
\hline $\begin{array}{l}\text { Cytochrome P450, Family 19, Subfamily A, } \\
\text { Polypeptide } 1\end{array}$ & cyp19a1 & 373656 & Rana_pipiens_Transcript_263865 & 3388 & BAA90529.1 & 0 & 837 \\
\hline Hydroxysteroid (17-beta) Dehydrogenase 12 & hsd17b12-b & 379747 & Rana_pipiens_Transcript_478585 & 1367 & NP_001080055.1 & $9.00 \mathrm{E}-87$ & 264 \\
\hline Estrogen Receptor 1 (alpha) & esr1-a & 398734 & Rana_pipiens_Transcript_057318 & 4278 & AAQ84782.1 & 0 & 924 \\
\hline Estrogen Receptor 2 (beta) & esr2 & 100174814 & Rana_pipiens_Transcript_334780 & 2192 & NP_001124426.1 & 0 & 917 \\
\hline Glucocorticoid Receptor & $\mathrm{nr} 3 \mathrm{c} 1-\mathrm{a}$ & 378598 & Rana_pipiens_Transcript_449022 & 6500 & CAA54804.1 & 0 & 589 \\
\hline $\begin{array}{l}\text { Hydroxy-Delta-5-Steroid Dehydrogenase, } 3 \\
\text { Beta- and Steroid Delta-Isomerase } 1\end{array}$ & hsd3b1 & 734818 & Rana_pipiens_Transcript_284246 & 2143 & NP_001089754.1 & 0 & 536 \\
\hline Hypoxia Inducible Factor 1 Alpha & hif-1a & 445838 & Rana_pipiens_Transcript_190140 & 4052 & ABF71072.1 & 0 & 1264 \\
\hline Steroid 11-Beta-Hydroxylase Protein & - & - & Rana_pipiens_Transcript_141896 & 1464 & AAQ04666.1 & $3.00 \mathrm{E}-152$ & 431 \\
\hline Steroidogenic Acute Regulatory Protein & star & 100381120 & Rana_pipiens_Transcript_388250 & 3776 & NP_001167502.1 & $2.00 \mathrm{E}-168$ & 497 \\
\hline Vitellogenin & vtga2 & 100037071 & Rana_pipiens_Transcript_478090 & 5777 & NP_001152753.1 & 0 & 1126 \\
\hline
\end{tabular}

To aid in the use of R. pipiens as a sentinel organism, putative R. pipiens endocrine-related genes were identified and the top BLAST hits are displayed.

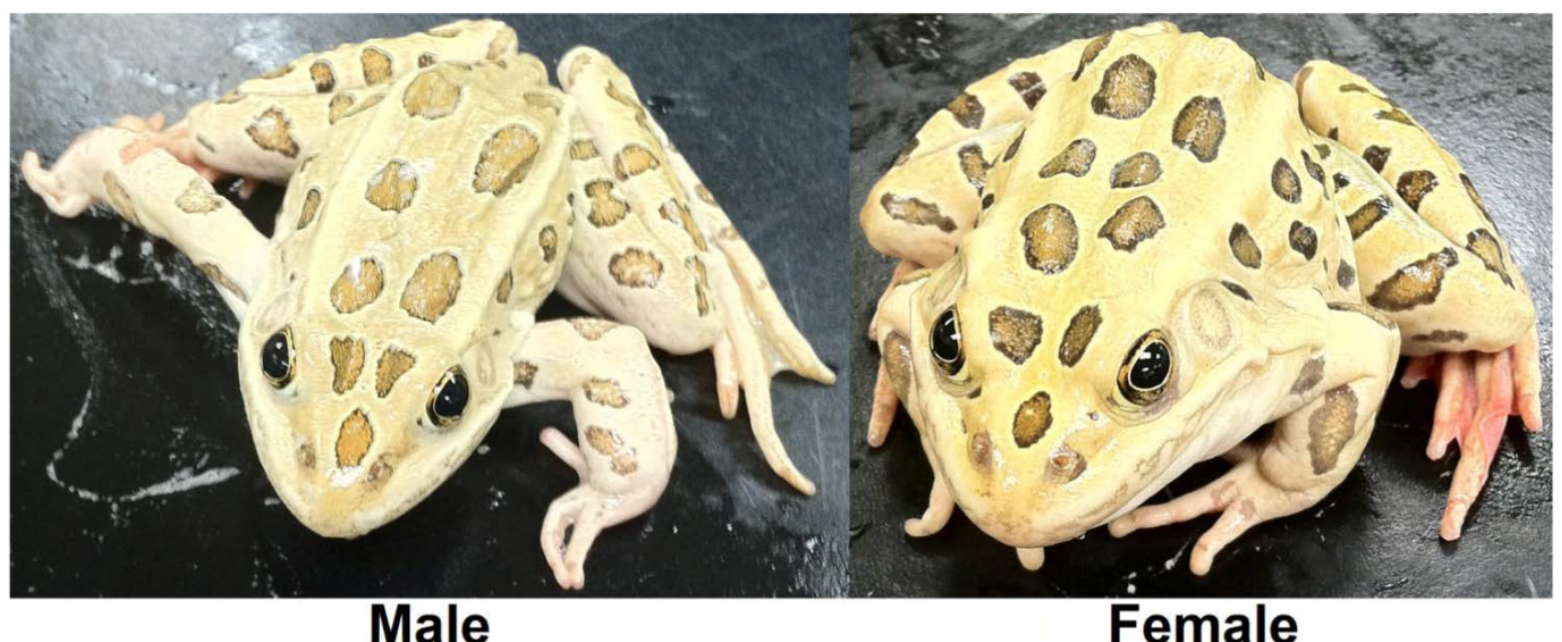

Figure I. The northern leopard frog Rana pipiens. From March to June mature R. pipiens gather at communal breeding ponds. Each female lays between 2,000 to 6,500 small, black and white eggs that hatch after two to three weeks [I]. Tadpoles are greenish or brown, with yellow or black speckles and their bellies are white and somewhat transparent, reaching $84 \mathrm{~mm}$ in length [1]. Metamorphosis typically occurs after 60 to 80 days, depending on conditions, and froglets are 20 to $30 \mathrm{~mm}$ long at metamorphosis [I]. Sexual maturity is reached in one to three years and adult $R$. pipiens are slender, long-legged green or brown with a white or cream underside, prominent, light-colored dorsolateral ridges, and large, dark spots located on its back, sides, and legs and grow to an average length of $68 \mathrm{~mm}$ and mass of $38.0 \mathrm{~g}[\mathrm{l}]$. 


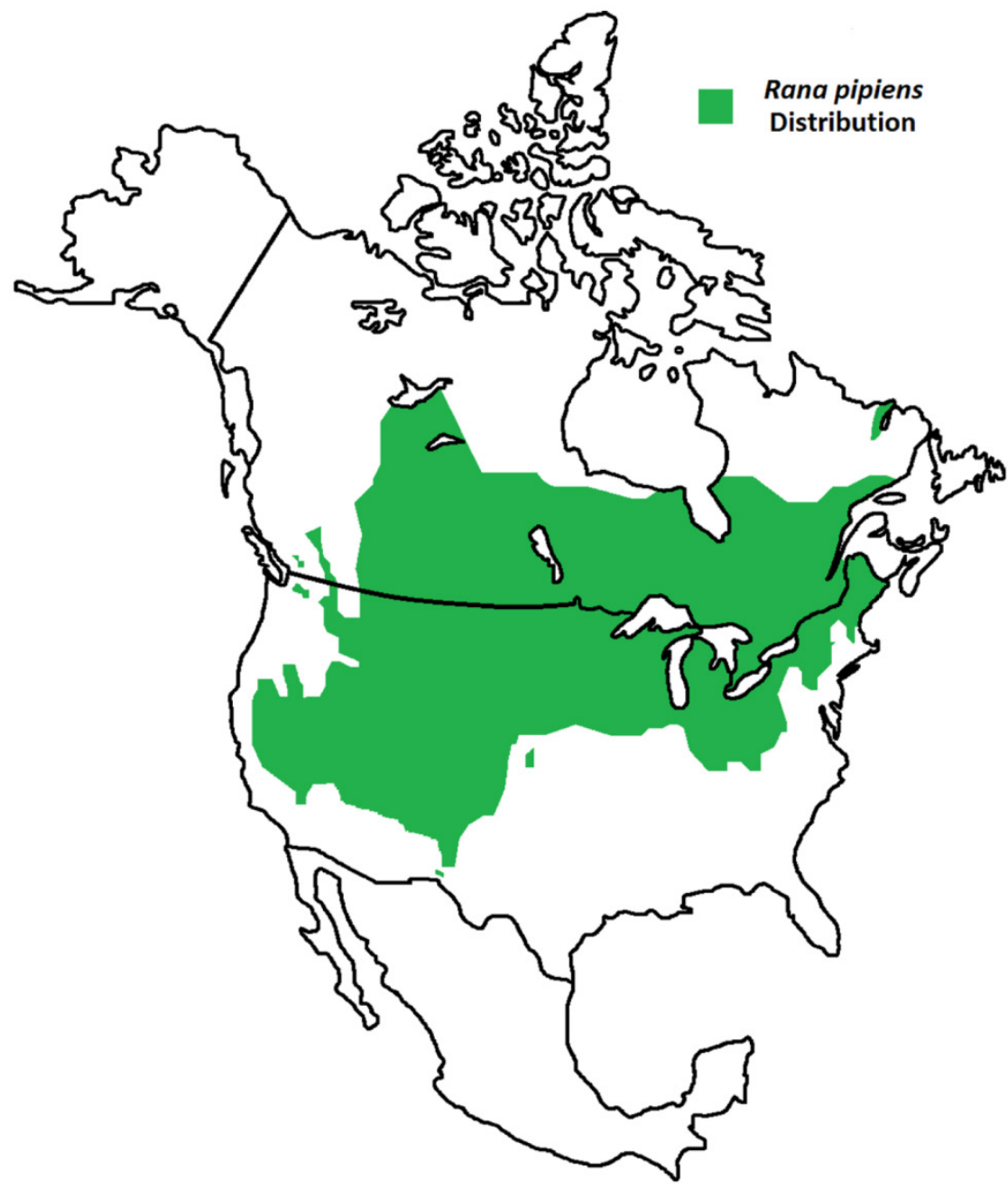

Figure 2. Geographic Distribution of Rana pipiens. R. pipiens is a native North American frog whose range extends from northern Canada to the southwest United States [I]. Its life cycle includes an aquatic larval stage and semi-terrestrial juvenile and adult stages; thus, this frog is found residing within grassland, brushland, and forest environments, prefering static or slow-moving water [I].

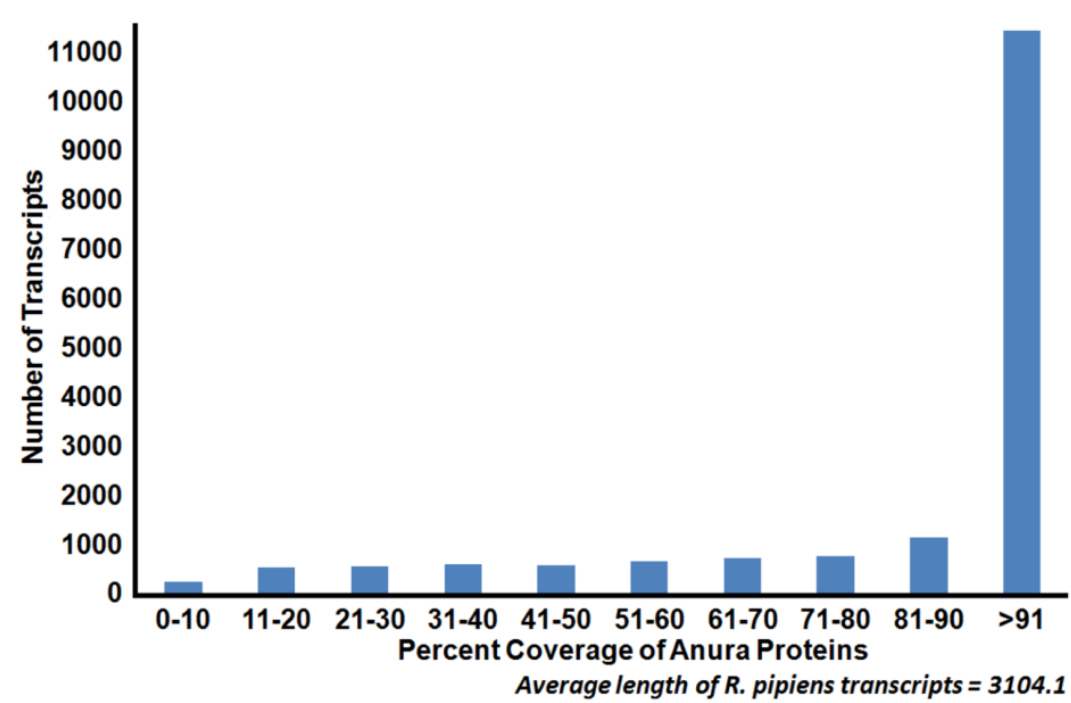

Figure 3. Coverage of Anura (frog) proteins by Rana pipiens transcripts. Sequence coverage length of $R$. pipiens transcripts when compared to available Anura (frog) proteins. The generated representative $R$. pipiens transcriptome was BLASTed against all available Anura proteins (count=159,284). The length of sequence homology as reported by BLASTx was compared to the length of the Anura protein homolog. The majority of the $R$. pipiens transcripts (45\%) had sequence homology with one or more Anura transcripts which covered at least $90 \%$ of the length of the Anura protein. 


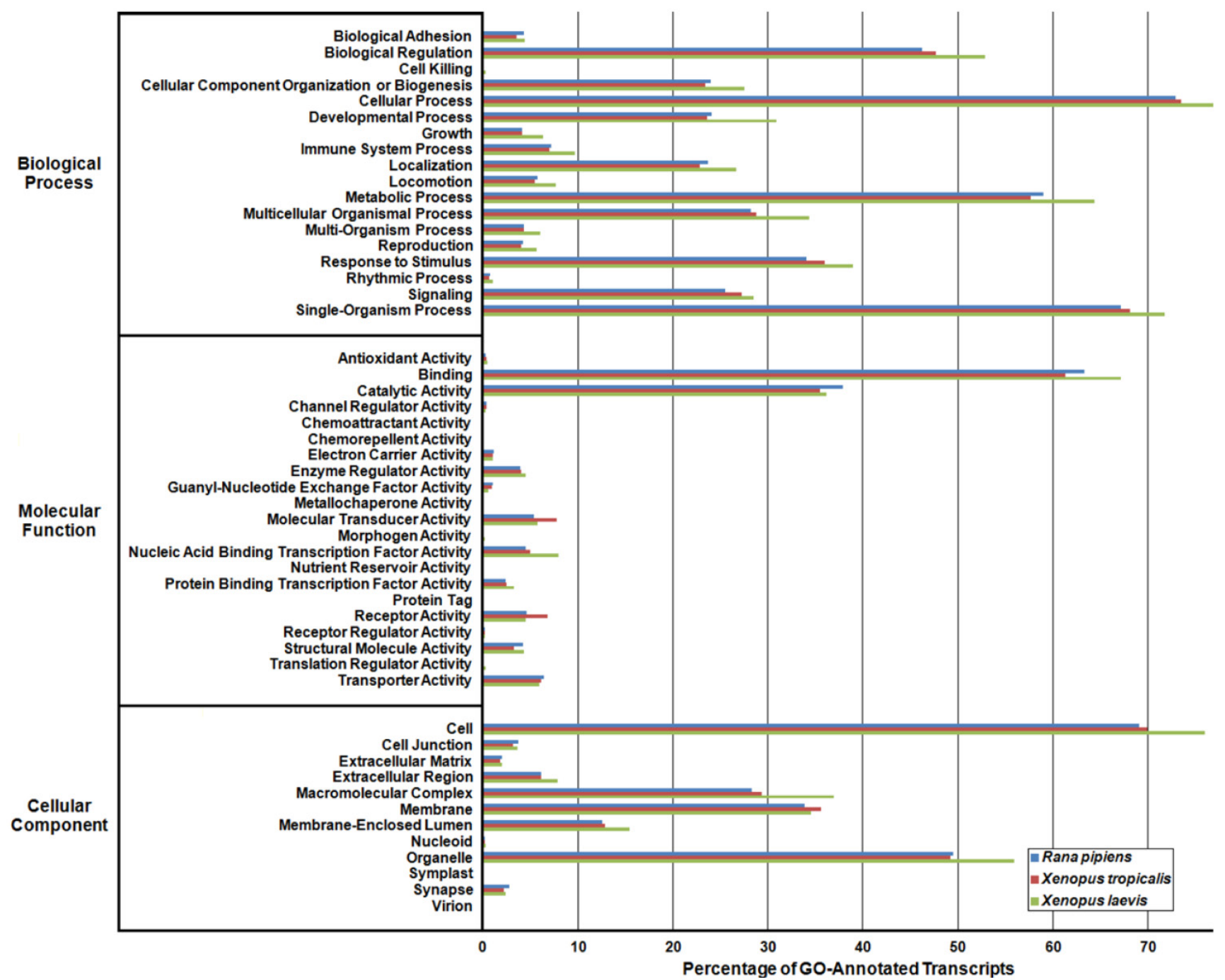

Figure 4. Gene Ontology (GO) Analysis of the Rana pipiens Transcripts. GO functional analysis was performed to evaluate transcript function and transcriptome completeness. GO terms were assigned to the R. pipiens transcripts and the mRNA RefSeq nucleotide entries of Xenopus tropicalis and Xenopus laevis retrieved from NCBI. The distributions of three transcriptomes closely resemble one another, suggesting the completeness of the $R$. pipiens transcriptome. 


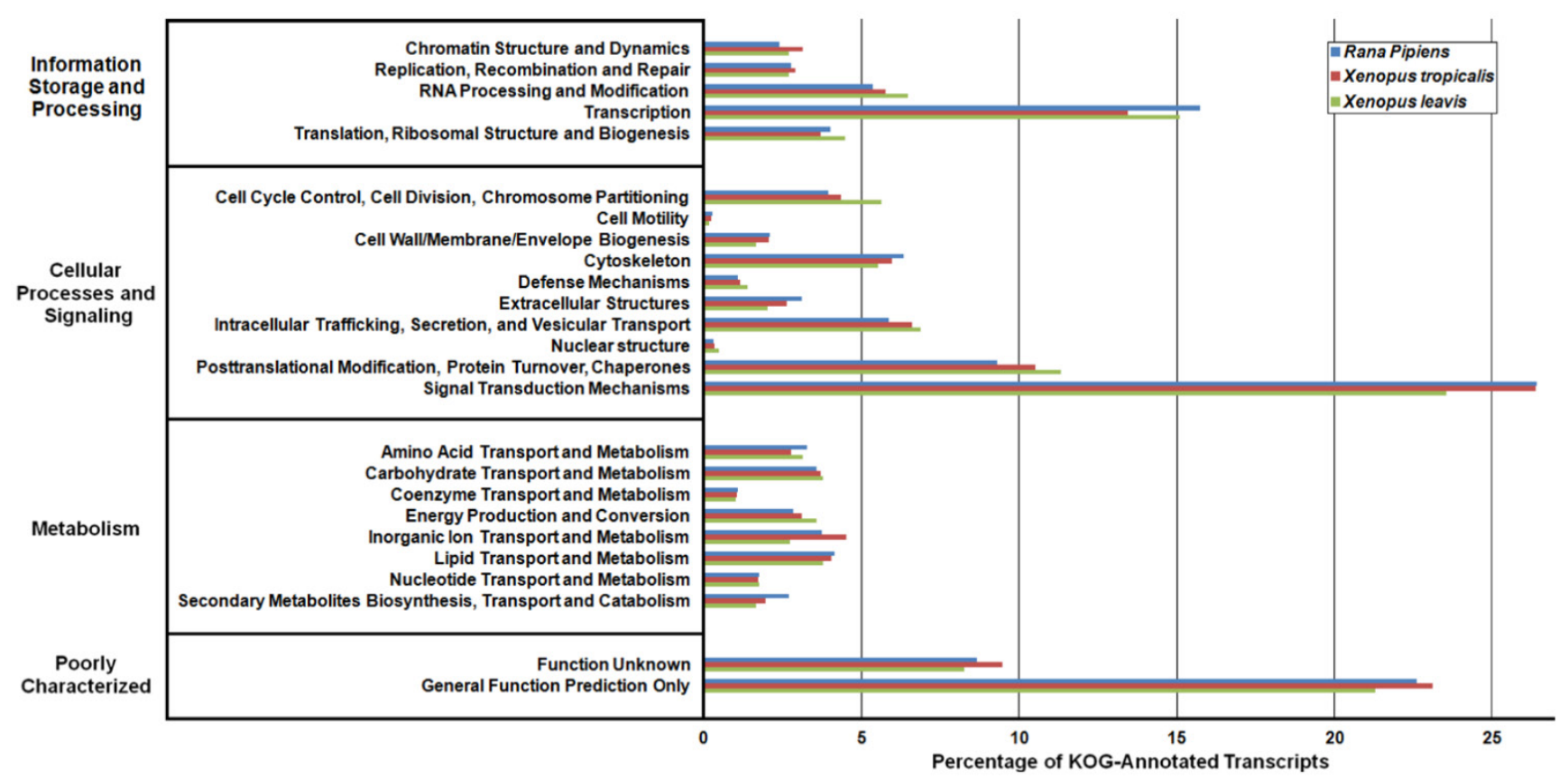

Figure 5. Eukaryotic Orthologous Groups (KOG) Characterization of Rana pipiens Transcripts. Putative transcript functions were assessed and transcriptome completeness was evaluated using KOG analysis. The R. pipiens transcriptome and mRNA nucleotide entries from NCBI of Xenopus tropicalis and Xenopus laevis were assigned KOG terms. The three transcriptomes have similar distributions, supporting the completeness of the R. pipiens transcriptome.

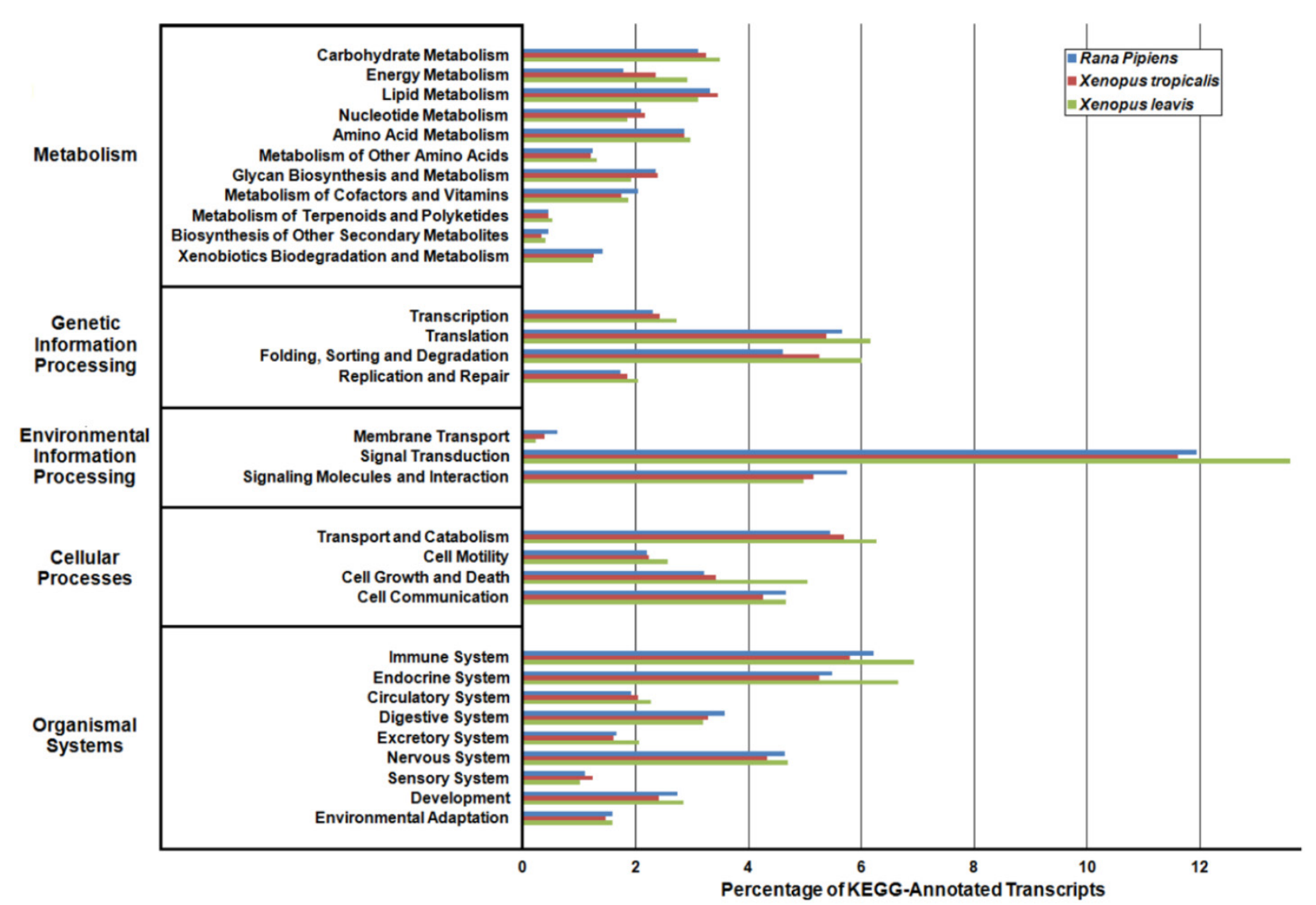

Figure 6. Kyoto Encyclopedia of Genes and Genomes (KEGG) Classification of Rana pipiens Transcripts. To review the putative functions of the transcripts and to assess the completeness of the transcriptome, KEGG analysis was performed. The $R$. pipiens transcripts and the mRNA nucleotide entries of Xenopus tropicalis and Xenopus laevis, retrieved from NCBI, were characterized by assigning K-numbers. The distributions of these three transcriptomes closely mimic one another, suggesting the completeness of the $R$. pipiens transcriptome. 


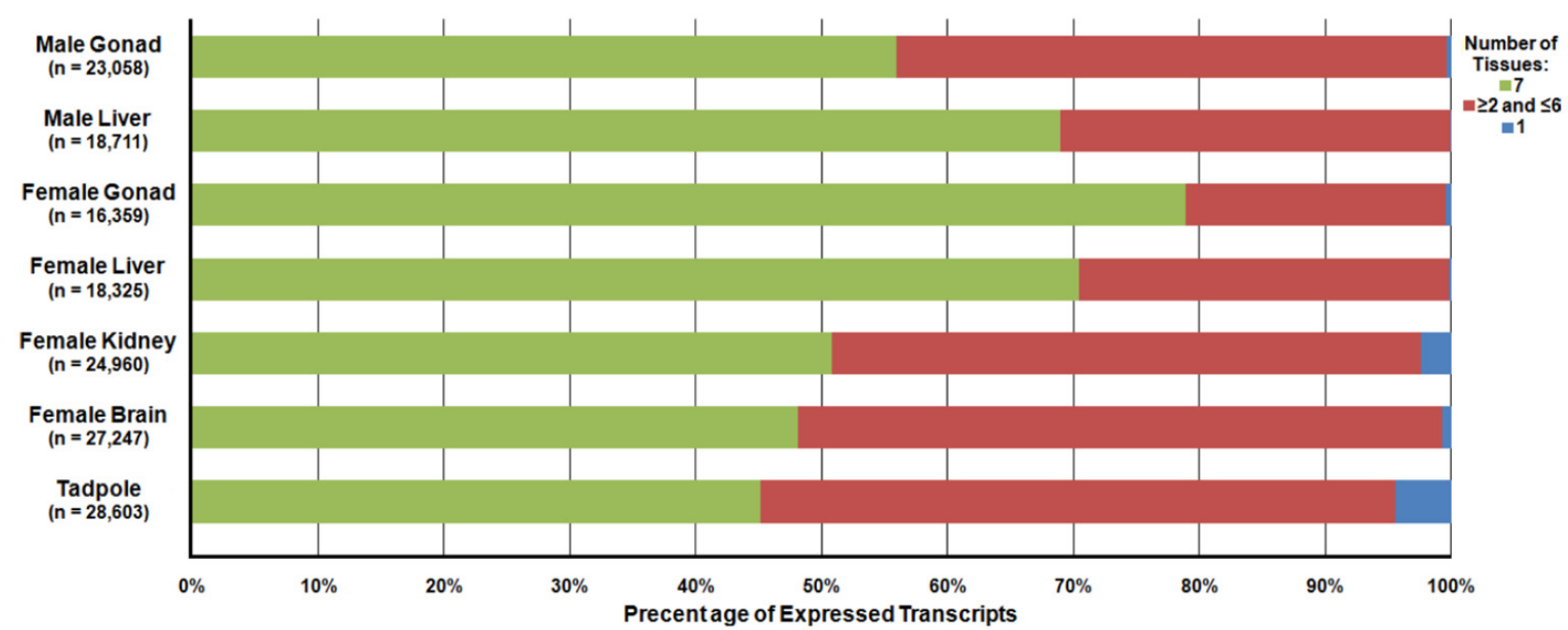

Figure 7. Differential Expression of Transcripts Present in Separate Rana pipiens Tissues. Differential expression analysis was performed to determine the abundance of the transcripts within each $R$. pipiens tissue. In total, 23,058, 18,7II, 16,359, 18,325, 24,960, 27,247, and 28,603 transcripts were detected and $72,12,76,33,182,612$, and I, 272 transcripts were unique to only that tissue (blue) in the male gonad, male liver, female gonad, female liver, female kidney, female brain, and tadpole, respectively. Moreover, 12,904 transcripts were present in all seven tissues (green) and a varied number of transcripts were present in more than one, but less than six, other tissues (red).

\section{Methods}

\section{Organism Growth Conditions, RNA Sample Preparation and Generation of Sequence Data}

Adult frogs and tadpoles were obtained from Science Kit \& Boreal Laboratories, Rochester, NY (Item Number 67496-32 and 67040-12, wild-caught). Adult $R$. pipiens were sacrificed and their organs, including gonad, liver, kidney, and brain, were isolated according to approved IUCAC protocols. Upon their arrival, the tadpoles were housed overnight (Group 1) and for one week (Group 2) within a 40 gallon tank. Following each period, the tadpoles were starved and placed into a tetracycline $(100 \mathrm{mg} / \mathrm{L})$ solution for 24 hours then sacrificed in accordance with approved IUCAC protocols. To preserve the integrity of the RNA, the isolated tissues were stored in RNAlater (Qiagen) for 24 hours at $4^{\circ} \mathrm{C}$ then placed at $-80^{\circ} \mathrm{C}$.

Total RNA was extracted from each tissue individually, using the RNeasy Plus Mini Kit (Qiagen). mRNA was purified from the total RNA preparations, using poly-T oligo-attached magnetic beads and converted into cDNA with random primers using the TruSeq ${ }^{\circledR}$ RNA Sample Preparation Kit v2 (Illumina). Libraries were sequenced using paired-end $100 \mathrm{bp}$ reads with an Illumina HiSeq 2000 sequence analyzer at the University of Nebraska Medical Center.

\section{Transcriptome Assembly}

To facilitate an accurate transcriptome assembly, reads were processed using PRINSEQ (version 0.17.3 lite) then Khmer (version 181e441) [7,8]. The pro- cessed reads were then assembled using Velvet (version 1.2.07) and Oases (version 1.2.07) in multi-k fashion $[2,3]$. Putative coding transcripts were identified in the redundant transcriptome by removing any transcript less than 200 bp with PRINSEQ, translating with TransDecoder (release Jan 16, 2014), reducing redundancy of the predicted proteins with CD-HIT and simplicity with PRINSEQ (version 0.17 .3 lite), and then using blat (version $35 \times 1$ ) to compare against UniProt (release 2014_02) and the X. tropicalis NCBI proteome (retrieved April 1, 2014). Potential non-coding RNAs were identified using blastn (E-value $\leq 1 \mathrm{e}-5)$ and the Rfam (version 11.0) and NONCODE (version 3.0) databases $[9,10]$. Transcripts that closely matched a UniProt or X. tropicalis protein or non-coding RNA were counted and in the case of multiple query matches to a single subject, the transcript with the highest bit-score was selected. The command-line codes used for transcriptome assembly are available at http://www.davislab.net/rana/.

\section{Similarity of Rana pipiens Transcripts to Other Species}

Transcripts were analyzed for sequence similarity by scanning the NCBI $\mathrm{nr}$ database (retrieved March 30, 2014) using blastx (E-value $\leq 1 \mathrm{e}-5)$. The top-hit species for each BLAST query was counted using Blast2GO (version 2.7.1) [11]. Representative transcripts were compared to Anura proteins (retrieved August 13, 2014) to determine their coverage using blastx ( $E$-value $\leq 1 \mathrm{e}-5)$ and MuSeqBox [12]. 


\section{Percent Identity Matrix Calculation}

Sequences obtained from the current assembly were aligned with sequences previously published to evaluate phylogenic relationships within amphibians [4]. Alignments were trimmed with Gblocks, concatenated into a single ordered sequence, and ClustalW alignment used to produce a percent identity matix $[13,14]$.

\section{Gene Ontology (GO), Eukaryotic Orthologous Groups (KOG), and Kyoto Encyclopedia of Genes and Genomes (KEGG)}

Multiple functional analyses were performed to assess the putative functions of the $R$. pipiens transcripts and to validate the transcriptome completeness, with $X$. tropicalis and $X$. laevis mRNA nucleotide entries from NCBI (retrieved April 1, 2014). First, blastx ( $E$-value $\leq 1 \mathrm{e}-5)$ was used to scan the transcriptome against the NCBI $\mathrm{nr}$ database (retrieved March 30, 2014) and GO terms were assigned using B2G4Pipe (version 2.5.0) and Blast2GO (version 2.7.1) with the b2g_may13 GO database [11]. Next, transcripts were translated by OrfPredictor (version 2.3) and these results were aligned using rps-blast $(E$-value of $\leq 1 \mathrm{e}-5)$ to the NCBI KOG database (version 3.0) $[15,16]$. Lastly, transcripts were compared to the Eukaryotic and Amphibian GENES datasets using the KEGG Automatic Annotation Server (version 1.68x) [17].

\section{Expression Profile of the Rana pipiens Tissues}

To calculate differential expression of transcripts between tissues, reads from each tissue were aligned to the transcriptome with Bowtie (version 2.2.2) and TopHat (version 2.0.11), and then Cuffdiff (version 2.2.0) was used to estimate transcript abundance $[18,19,20]$.

\section{Identification of Endocrine-Related Genes}

Endocrine-related genes present within the $R$. pipiens transcriptome were identified by comparing the transcriptome to a custom BLAST database containing endocrine-related genes from $X$. laevis (retrieved from NCBI on October 8, 2013) using blastx $(E$-value $\leq 1 \mathrm{e}-5)$.

\section{Acknowledgements}

Financial support for the present study was provided by grants from the Nebraska Academy of Sciences and the Minnesota Herpetological Society. The University of Nebraska provided additional support through the Biomedical Research Training Program, the Fund for Investing in the Research Enterprise, the Graduate Research and Creative Activity
Fund, the Holland Computing Center, and the Fund for Undergraduate Scholarly Experience.

The following NIH awards supported this work: NCRR RR027754, RR016469-8348, RR018788-08 and NIGMS GM103427, GM103471. This publication's contents are the sole responsibility of the authors and do not necessarily represent the official views of the NIH.

\section{Competing Interests}

The authors have declared that no competing interest exists.

\section{References}

1. [Internet] US Environmental Protection Agency. Species Profile: Northern Leopard Frog. http://www.epa.gov/housatonic/thesite/restofriver/reports/final_era /B\%20-\%20Focus\%20Species\%20Profiles/EcoRiskProfile_leopard_frog. pdf

2. Zerbino DR, Birney E. Velvet: algorithms for de novo short read assembly using de Bruijn graphs. Genome Res. 2008;18:821-829

3. Schulz MH, Zerbino DR, Vingron M, Birney E. Oases: robust de novo RNA-seq assembly across the dynamic range of expression levels. Bioinformatics. 2012;28:1086-1092

4. Frost DR, Grant T, Faivovich J, Bain RH, Haas A, Haddad CFB, de Sá RO, Channing A, Wilkinson M, Donnellan SC, Raxworthy CJ, Campbell JA, Blotto BL, Moler P, Drewes RC, Nussbaum RA, Lynch JD, Green DM, Wheeler WC. The Amphibian Tree of Life. Bull Am Mus Nat Hist. 2006;297:1-370

5. Knight LA, Christenson MK, Trease AJ, Davis PH, Kolok AS. The spring runoff in Nebraska's (USA) Elkhorn River watershed and its impact on two sentinel organisms. Environ Toxicol Chem. 2013;32:1544-1551

6. Hayes TB, Anderson LL, Beasley VR, de Solla SR, Iguchi T, Ingraham H, Kestemont P, Kniewald J, Kniewald Z, Langlois VS, Luque EH, McCoy KA, Muñoz-de-Toro M, Oka T, Oliveira CA, Orton F, Ruby S, Suzawa M, Tavera-Mendoza LE, Trudeau VL, Victor-Costa AB, Willingham E. Demasculinization and feminization of male gonads by atrazine: consistent effects across vertebrate classes. J Steroid Biochem Mol Biol. 2011;127:64-73

7. Schmieder R, Edwards R. Quality control and preprocessing of metagenomic datasets. Bioinformatics. 2011;27:863-864

8. [Internet] Brown CT, Howe A, Zhang Q, Pyrkosz AB, Brom TH. A Reference-free algorithm for computational normalization of shotgun sequencing data. http://arxiv.org/abs/1203.4802v2

9. Burge SW, Daub J, Eberhardt R, Tate J, Barquist L, Nawrocki EP, Eddy SR, Gardner PP, Bateman A. Rfam 11.0: 10 years of RNA families. Nucleic Acids Res. 2013;41:226-232

10. Bu D, Yu K, Sun S, Xie C, Skogerbø G, Miao R, Xiao H, Liao Q, Luo H, Zhao G, Zhao H, Liu Z, Liu C, Chen R, Zhao Y. NONCODE v3.0: integrative annotation of long noncoding RNAs. Nucleic Acids Res. 2012;40:210-215

11. Conesa A, Götz S, García-Gómez JM, Terol J, Talón M, Robles M. Blast2GO: a universal tool for annotation, visualization and analysis in functional genomics research. Bioinformatics. 2005;21:3674-3676

12. Xing L, Brendel V. MuSeqBox: a program for multi-query sequence BLAST output examination. Bioinformatics. 2001;17:744-745.

13. Larkin MA, Blackshields G, Brown NP, Chenna R, McGettigan PA, McWilliam H, Valentin F, Wallace IM, Wilm A, Lopez R, Thompson JD, Gibson TJ, Higgins DG. Clustal W and Clustal $X$ version 2.0. Bioinformatics. 2007;23:2947-2948

14. Castresana J. Selection of conserved blocks from multiple alignments for their use in phylogenetic analysis. Mol Biol Evol. 2000;17:540-552

15. Min XJ, Butler G, Storms R, Tsang A. OrfPredictor: predicting protein-coding regions in EST-derived sequences. Nucleic Acids Res. 2005;33:677-680.

16. Tatusov RL, Fedorova ND, Jackson JD, Jacobs AR, Kiryutin B, Koonin EV, Krylov DM, Mazumder R, Mekhedov SL, Nikolskaya AN, Rao BS, Smirnov S, Sverdlov AV, Vasudevan S, Wolf YI, Yin JJ, Natale DA. The COG database: an updated version includes eukaryotes. BMC Bioinformatics. 2003;4:1-14. 
17. Moriya $\mathrm{Y}$, Itoh M, Okuda S, Yoshizawa AC, Kanehisa M. KAAS: an automatic genome annotation and pathway reconstruction server. Nucleic Acids Res. 2007;35:182-185.

18. Langmead B, Salzberg S. Fast gapped-read alignment with Bowtie 2. Nat Methods. 2012;9:357-359

19. Kim D, Pertea G, Trapnell C, Pimentel H, Kelley R, Salzberg SL. TopHat2: accurate alignment of transcriptomes in the presence of insertions, deletions and gene fusions. Genome Biol. 2013;14:1-13

20. Trapnell C, Williams BA, Pertea G, Mortazavi A, Kwan G, van Baren MJ, Salzberg SL, Wold BJ, Pachter L. Transcript assembly and quantification by RNA-Seq reveals unannotated transcripts and isoform switching during cell differentiation. Nat Biotechnol. 2010;28:511-515. 